\title{
OBSERVACIONES HIDROGRAFICAS EN BAHIA FOSTER Y BAHIA CHILE (ISLAS SHETLAND DEL SUR) ENERO 1978
}

DAGOBERTO ARCOS R. Y MARCO A. SALAMANCA O.

Departamento de Biología Marina y Oceanografía, Instituto de Biología, Universidade de Concepción Concepción, Chile

\section{SYNOPSIS}

The hydrographical conditions founded in Port Foster (Deception Island) and Chile Bay (Greenwich Island) both belong to the South Shetlands Islands group. Antarctic Peninsula, has been described. This survey has been carried out from the Chilean Navy Oceanographic vessel "YELCHO" in January 1978. An stratification in the area closed with the Antarctic surface waters has been observed. The temperature ranged between - 1.60 $\mathrm{O}_{\mathrm{C}}$ to $1,30^{\circ} \mathrm{C}$ in Port Foster and $0,46^{\circ} \mathrm{C}$ to $1,70^{\circ} \mathrm{C}$ in Chile Bay; the salinity ranged between $33.80 \%$ to $34,24 \%$ in Port Foster and $33,61 \%$ to $34,10 \%$ in Chile Bay; the dissolved oxygen varied between 3.47 to $7,02 \mathrm{ml} 02 / \mathrm{l}$ in both area. The characteristic density (Sigma t) of Antarctic Surface waters in the area, i.e., 26,92 and 27,58 has been observed.

\section{Introduccion}

En 1967. la Universidad de Concepción y el Instituto Antártico Chileno iniciaron el estudio biológico de la fauna béntica planctónica en algunas Bahías Antárticas en el sector de las Islas Shetland del Sur. Ese mismo año (diciembre de 1967 y enero de 1968 ) ocurrieron grandes erupciones volcánicas en Bahía Foster. Isla Decepción ( $62^{\circ} 59^{\prime} \mathrm{S}$ y $\left.60^{\circ} 35^{\prime} \mathrm{W}\right)$ afectando a la fauna béntica. Nuevas erupciones se observaron en 1969 y 1970 . Los estudios bénticos continuaron con el objeto de estudiar las etapas de repoblación de la fauna de Bahía Foster. Como punto de comparación se utilizó a Bahía Chile, Isla Greenwich $\left(62^{\circ} 29^{\prime} \mathrm{S}\right.$ y $\left.59^{\circ} 38^{\prime} \mathrm{W}\right)$ como zona inalterada (Gallardo et al., 1968, 1969, 1970 y 1977).

El presente trabajo informa de las condiciones hidrográficas encontradas en Bahía Foster, Isla Decepción y Bahía Chile, Isla Greenwich, en enero de 1978, como complemento a los estudios bénticos y planctónicos allí realizados

La XXXII Expedición Antártica, se realizó a bordo del AGS YELCHO, buque oceanográfico de la Armada de Chile, efectuando muestreos hidrográficos entre los días 12 al 15 de enero de 1978 en Bahía Foster, Isla Decepción; 16 - 17 en Bahía Chile, Isla Greenwich, ambas situadas en el grupo de las Islas Shetland del Sur (Fig. 1).

Se utilizó 11 estaciones de muestreo en Bahía Foster y 6 estaciones en Bahía Chile. En cada una de ellas se observó, mediante el uso de botellas Nansen y termómetros de inversión protegidos. la temperatura, la salinidad y el contenido de oxígeno disuelto en el agua de mar a diferentes profundidades.

La temperatura fue medida con termómetros de inversión protegidos Kurt Gohla $\left(-2^{\circ} \mathrm{C}\right.$ a $\left.+33^{\circ} \mathrm{C}\right)$ y la salinidad medida con un salinómetro de inducción Autolab MK III El oxígeno disuelto fue determinado siguiendo el método de Winker modificado por Carritt \& Carpenter (1966). Cálculos de densidad expresados como sigma $t$ fueron hechos para todos los valores y se dibujó secciones transversales de temperatura y salinidad para las dos áreas en estudio.

\section{Resultados}

BAHLA FOSTER

La distribución vertical de temperatura presentó una clara tendencia a la estratificación (Fig. 2) variando entre $1,30^{\circ} \mathrm{C}$ cercano al fondo.

Se observa un aumento de la temperatura superficial hacia el interior de la Bahía desde la Est. 22 con valores entre 0,2 y $1,3^{\circ} \mathrm{C}$ $\mathrm{Se}$ insinúa un sector con valores sobre $1,0^{\circ} \mathrm{C}$ en las estaciones cercanas al fondo de saco de la Bahía (Fig. 2)

Por otro lado, la salinidad presenta una estratificación, observando-se valores entre $33.80 \%$ y $34.24 \%$, un núcleo de agua superficial con valores sobre $33,90 \%$ en las estaciones 2 y 25 y "lente" de agua con valores sobre 34,00 \%o cercana a la superficie (2 y $10 \mathrm{~m}$ ) en la estación 24 . Valores de $34,10 \%$ se observó en toda la Bahía desde los $60 \mathrm{~m}$ de profundidad y el fondo (Fig. 3).

El oxígeno disuelto se presentó muy estable con valores entre $3,47 \mathrm{ml} \mathrm{O} 2 / \ell$ y $7,09 \mathrm{ml} \mathrm{O}_{2} / \ell$. Los valores de sigma $t$ fueron estrechos con poca variación entre 27,10 y 27.58 .

Los parametros anteriores se encuentran dentro del rango de variación para las aguas superficiales antárticas.

\section{BAHIA CHILE:}

La distribución vertical de la temperatura es claramente estratificada según lo muestra la Figura 4 , con valores sobre $0^{\circ} \mathrm{C}$ y hasta $1,70^{\circ} \mathrm{C}$

La salinidad, igual que la temperatura, es claramente estratificada presentado valores entre 33,61 y $34,10 \%$ (Fig. 5 ).

Se observa un núcleo de aguas con salinidades menores a $33,8 \%$ en la estación $3 \mathrm{y}$ a una profundidad de $5 \mathrm{~m}$. Esto se de bería a la presencia en ese sector de un glaciar que llega hasta e borde de la bahía y que en esta época del año (enero) se produce el deshielo.

El oxígeno disuelto presentó poca variación, entre 6,25 y $7,02 \mathrm{ml} \mathrm{O}_{2} / \ell$. Los valores de sigma $\mathrm{t}$ variaron muy poco, entre 26,92 y 27,37 .

De las observaciones anteriores se puede concluir que las aguas en Bahía Foster y Bahía Chile presentan tendencias a la estratificación. En ambas bahías se encuentran valores de temperatura y salinidad cercanos a los característicos para las Aguas Antárticas Superficiales (AAS) concordando con lo observado por Silva \& Muñoz (1975) para Bahia Foster.

Sigma t varió entre 26,92 y 27,58 siendo 27,00 el valor ti. pico para las AAS; en ambas bahías la columna de agua estaba bien oxigenada con un $87 \%$ de saturación máxima y temperaturas mínimas cercanas al punto de congelación, i.e.. $1.79^{\circ} \mathrm{C}$

En estas bahías puede encontrarse pequeños bolsones de agua con características diferentes $y$ cercanos a la costa como resultado de situaciones locales, i.e, Bahía Foster, temperaturas mas altas en los sectores cercanos a los afectados por el vulcanismo y que indudablemente afectan a los cuerpos de agua cirundantes; Bahía Chile, salinidades más bajas en sectores influenciados por la cercanía de glaciares.

\section{Agradecimientos}

Es nuestro especial interés reconocer el patrocinio y financiamiento del Instituto Antártico Chileno y agradecer a la tripulación del AGS YELCHO. Armada de Chile, sin cuya colaboración no habría sido posible realizar este trabajo. 


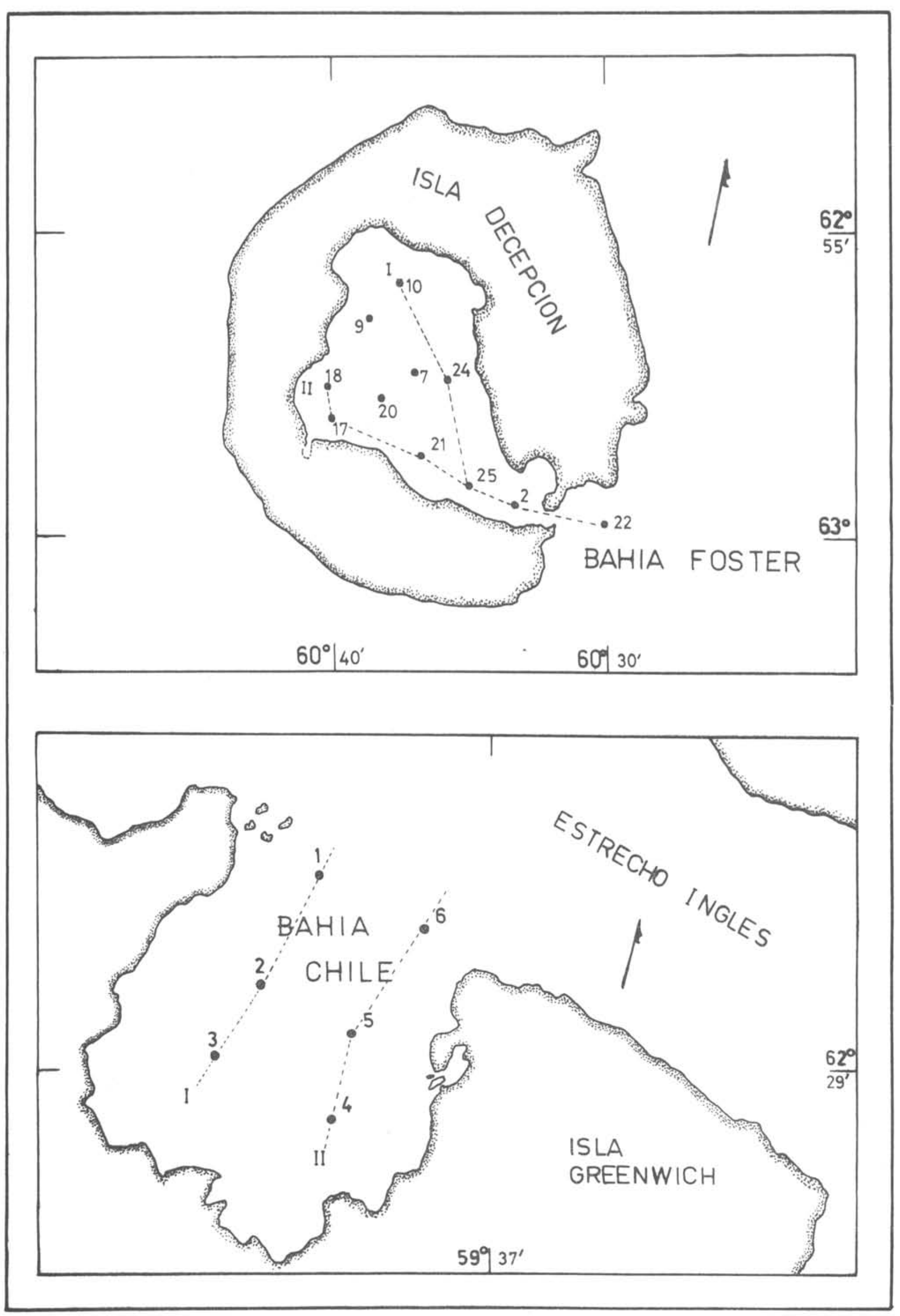

Fig. 1 - Bahía Foster y Bahía Chile, ubicación de los lugares de muestreos. 


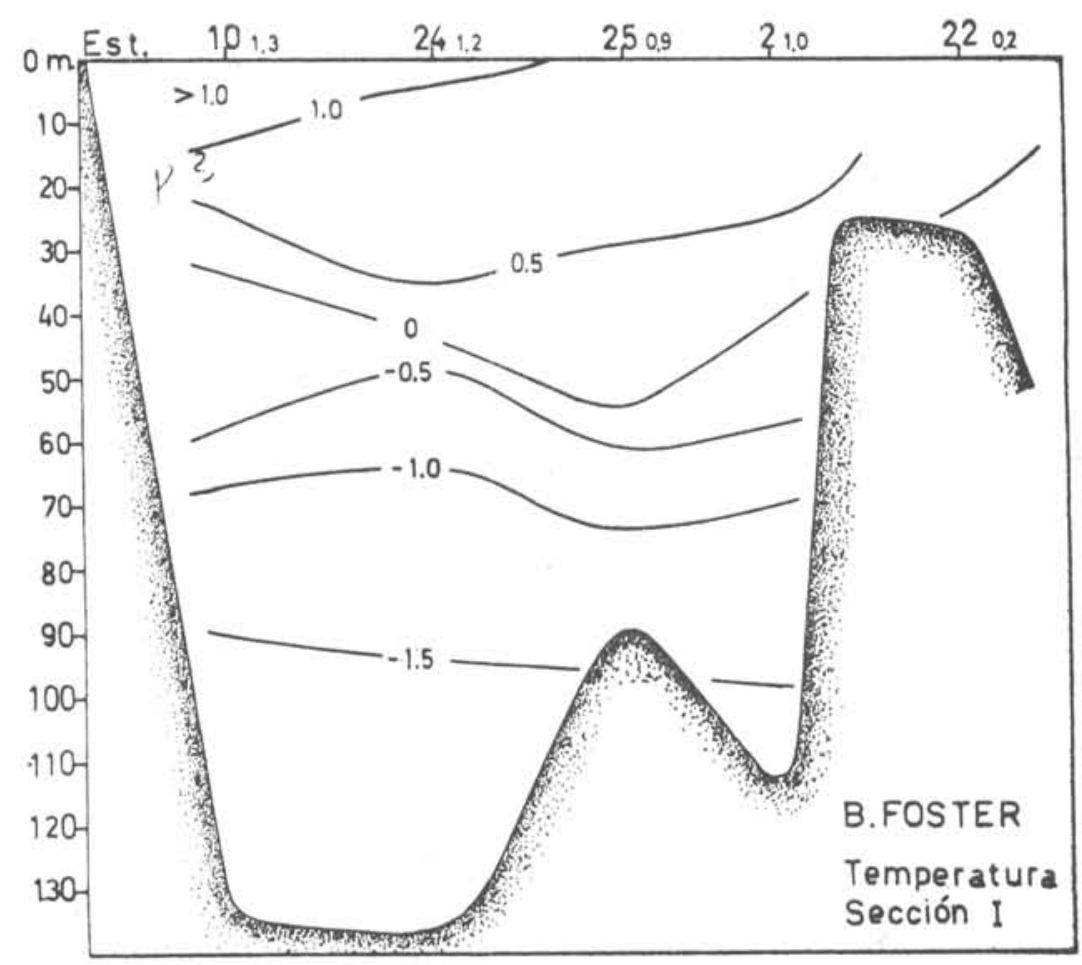

Fig. 2 - Distribución vertical de la temperatura en la sección 1 de Bahía Foster.

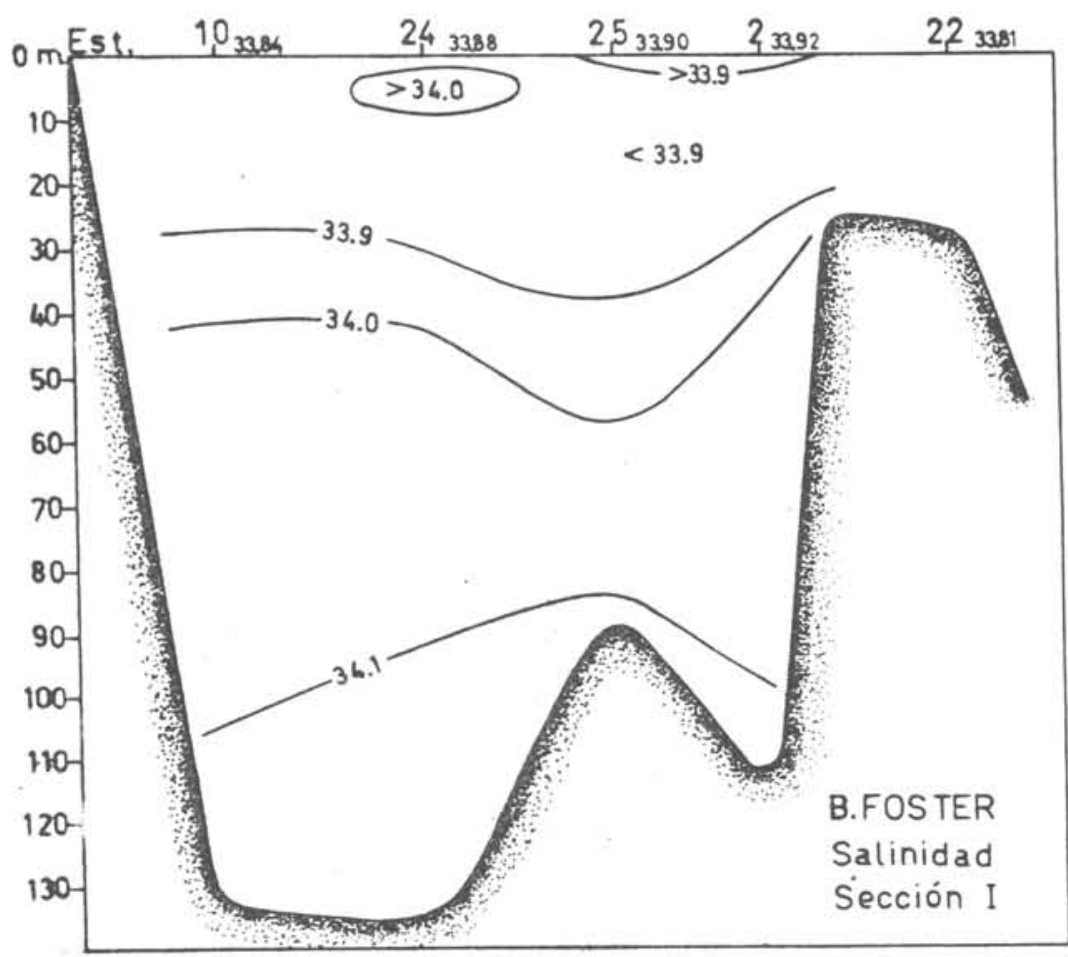

Fig. 3 - Distribución vertical de la salinidad en la sección I de Bahía Foster. 


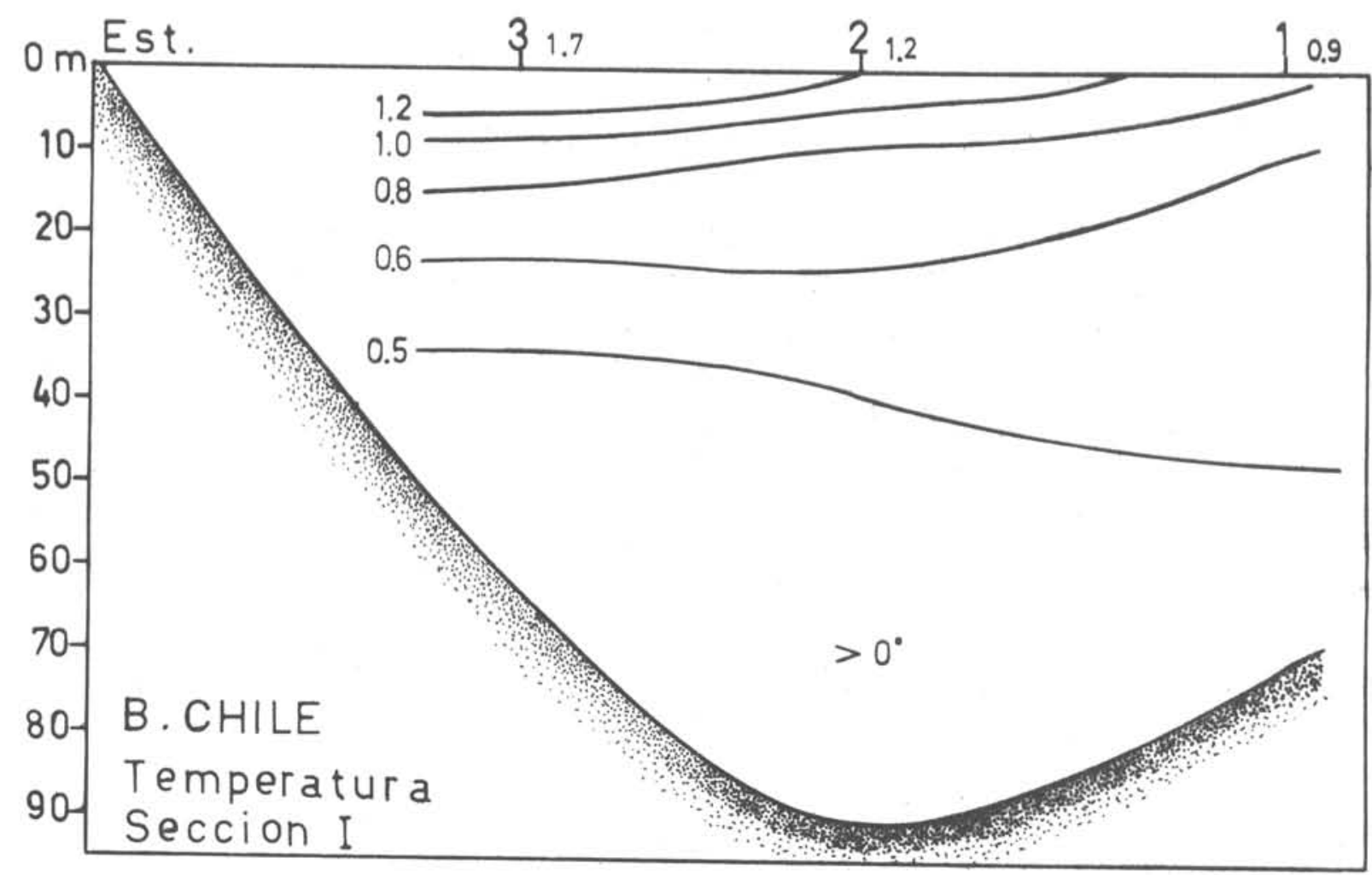

Fig. 4 - Distribución vertical de la temperatura en la sección I de Bahía Chile.

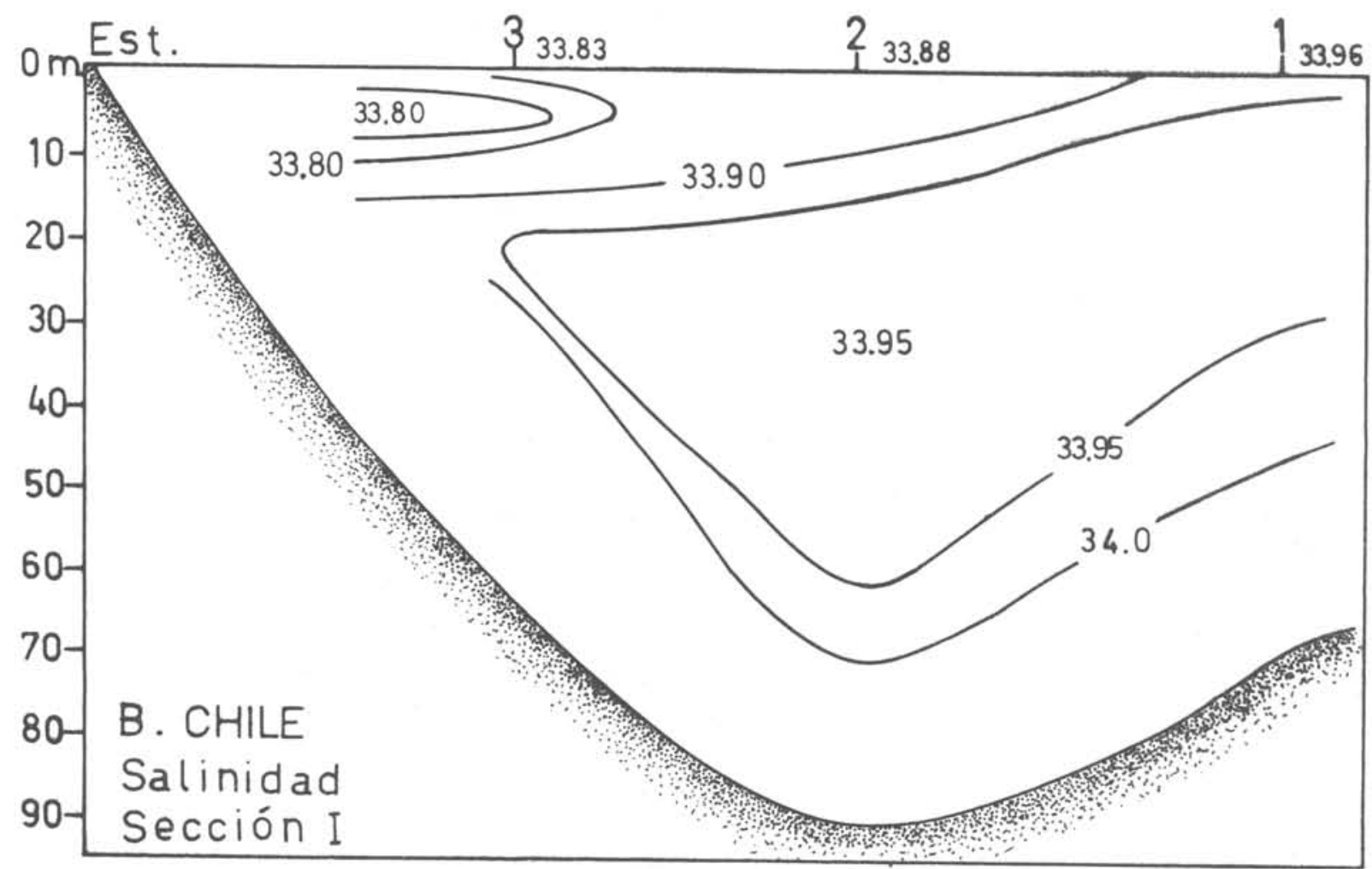

Fig. 5 - Distribución vertical de la salinidad en la sección I de Bahía Chile. 


\section{Referfncias Bibliograficas}

CARRITT, D.E. \& CARPENTER, J.H. 1966. Comparison and evaluation of currently employed modifications of Winkler method for determining dissolved oxygen in seawater: a NASCO report. J. mar. Res., 24 (3): 286-318.

GALLARDO, V.A. \& CASTILLO, J.G. 1968. Mass mortality in the benthic infauna of Port Foster resulting from the eruptions in Deception Island, South Shetland Is. Publnes Inst. antart. chileno, (16): 1-13. - \& $\&-n-1969$. Quantitative benthic survey of the infauna of Chile Bay (Greenwich Is., South Shetland Is.). Gayana, Zool., (16): 1-18.
-- \& - - - - 1970. Quantitative observations on the benthic macrofauna of Port Forter (Deception Island) and Chile Bay (Greenwich Island). In: Holdgate, M.W., ed. Antarctic ecology. London, Academic Press, vol. 1. p. 242 . 243.

; H.I. \& HERMOSILLA, J.G. 1077. Ouantitative studies on the softbottom macrobenthic animal communities of shallow Antarctic bays. In: Llano, G.A., ed. - Adaptation whrthin Antarctic ecosystem. Proc. Third SCAR Symp. on Antarctic Biology, p. 361-387.

SILVA, N. \& MUÑOZ, J. 1975. Descripción de las condiciones oceanográficas de la Bahía Foster, Isla Decepción. Enero de 1967. Serie cient. Inst. antart. chileno, 3(1): 80-86. 